\title{
Expression and Significance of Toll-Like Receptor 1 and Toll-Like Receptor 3 in Renal Tissues of Children with Primary Nephrotic Syndrome
}

\author{
XIN ZHENG, XIAOZHI YAO, Q. CHEN AND L. CHEN* \\ Department of Pediatric, Shengli Clinical Medical College, Fujian Medical University, Fujian Provincial Hospital South Branch, \\ FuZhou 350001, China
}

Zheng et al.: Expression of Toll-Like Receptor 1 and Toll-Like Receptor 3 in Renal Tissues

\begin{abstract}
To explore the expression and clinical significance of toll-like receptor 1 and toll-like receptor 3 in renal tissues of children with primary nephrotic syndrome. A total of 120 children with primary nephrotic syndrome kidney tissues who underwent renal biopsy were selected as the observation group, which can be divided into minimal change disease type (40 cases), membranous nephropathy type (20 cases), mesangial proliferative glomerulonephritis type (30 cases) and focal segmental glomerulosclerosis type (30 cases). In addition, the normal kidney tissues of 20 children with Wilms tumor admitted to the same period were selected as the control group and immunohistochemistry was used. The method was used to detect the expression levels of toll-like receptor 1 and toll-like receptor 3 in the kidney tissues of the two groups. Compared with the control group, the different pathological types in the observation group had higher expression levels of toll-like receptor 1 in renal tubular tissues. The expression levels of toll-like receptor 1 in minimal change disease type renal tubular tissues in different pathological types of tissues were significantly higher than those in other groups, followed by membranous nephropathy. In mesangial proliferative glomerulonephritis type and focal segmental glomerulosclerosis type, the difference was statistically significant (all $\mathbf{p}<\mathbf{0 . 0 5}$ ). Compared with the control group, the different pathological type groups in the observation group had higher expression levels of toll-like receptor 3 in renal tubular tissues. The expression levels of toll-like receptor 3 in mesangial proliferative glomerulonephritis type renal tubular tissues in different pathological types of tissues were significantly higher than those in other groups, followed by membranous nephropathy type, minimal change disease type and focal segmental glomerulosclerosis type, the difference was statistically significant (all $\mathbf{p}<0.05$ ). Toll-like receptor 1 and toll-like receptor 3 have high expression levels in the renal tissues of children with primary nephrotic syndrome and their expression levels have certain differences in different pathological types.
\end{abstract}

Key words: Toll-like receptor 1 , toll-like receptor 3, primary nephrotic syndrome, immunohistochemistry

Primary Nephrotic Syndrome (PNS) is the most common glomerular disease in childhood ${ }^{[1]}$. It has a long course and is easy to relapse. It is the main cause of chronic kidney failure in children. About $90 \%$ of children's PNS have unknown etiology. It is currently believed that its possible pathogenesis is related to the abnormal secretion of cytokines (especially Interleukin-1 (IL-1) and Tumor Necrosis Factor alpha $(\mathrm{TNF}-\alpha)$ ) caused by T lymphocyte dysfunction ${ }^{[2-4]}$. TollLike Receptors (TLRs) are important innate immune receptors discovered in recent years. They can recognize Pathogen-Associated Molecular Patterns (PAMPs) of pathogenic microorganisms and then couple with signal transduction. The pathway activates natural immune cells. At the same time, it can also provide costimulatory signals for activating adaptive immunity and ultimately cause a series of immune response-mediated inflammation. Toll-Like Receptor 1 (TLR1) in the family of TLRs that have been discovered, mainly recognizes bacterial Lipopolysaccharide (LPS) and

*Address for correspondence

E-mail: chenlang11@qq.com 
Toll-Like Receptor 3 (TLR3) mainly recognizes viral nucleic acids. Recent studies have found that TLR1 and TLR3 signaling pathways can mediate a variety of infectious and non-infectious kidney diseases ${ }^{[5,6]}$. In this paper, children who were diagnosed with PNS and confirmed renal pathology by renal biopsy were used as the research object. Immunohistochemical methods were used to detect the expression of TLR1 and TLR3 in renal tissues and analyze the relationship between them and different types of renal pathology. From January 2018 to January 2019, 110 children with PNS diagnosed by the Provincial Clinical College of Fujian Medical University and renal biopsy confirmed renal pathology was selected as the research objects. The inclusion criteria of the study subjects were: The age and sex of children with PNS were similar; Wilms tumor was diagnosed and had been operated on; No history of infection within 1 mo before the operation; complete clinical data. The clinical types of PNS include Minimal Change Disease (MCD), Focal Segmental Glomerulosclerosis (FSGS), Mesangial Proliferative Glomerulonephritis (MsPGN), Membranous Proliferative Glomerulonephritis (MPGN), Membranous Nephropathy (The standard of $\mathrm{MN}$ ) is based on the standard of the "Trial" draft of the guideline for diagnosis and treatment of hormoneresistant nephrotic syndrome. Kidney pathology reading and classification are based on kidney pathology sections by professionals engaged in renal pathology in our hospital for reading and diagnosis. Renal pathological classification was classified according to World Health Organization (WHO) 1982 and modified 1995 pathological classification standards of glomerular diseases. Kidney tissue extraction method is described below. Kidney puncture and renal tissue acquisition of PNS in children with a clear diagnosis and no contraindications to renal puncture and the children's family members signed a consent form to perform renal puncture. Under the guidance of B-ultrasound in a sterile room, a kidney puncture needle is used and the puncture point is the lower pole of the right kidney. In children with renal tumor resection, the kidney tissue is taken from the site far from the edge of the renal tumor and confirmed by light microscope. Immunohistochemistry is used to detect the expression of TLR1 and TLR3 in kidney tissue. The expression of TLR1 and TLR3 in kidney tissue was detected by immunohistochemical two-step method to detect the expression of TLR1 and TLR3 in kidney tissue. The operation steps were carried out according to the instructions of GTVisionTMIII anti-mouse/rabbit universal immunohistochemical detection kit (Shanghai Soleb Biotech Co., Ltd.) The working concentration of TLR1 and TLR3 (both purchased from R\&D Company in the United States) is 1:50, 3,3'-Diaminobenzidine (DAB) color development (Shanghai Soleb Biotech Co., Ltd.) and hematoxylin counterstain. The evaluation of TLR1 and TLR3 immunohistochemistry was carried out using IDA-2000 high-definition digital microscope image analysis system. The tan stained area in the kidney tissue was taken as the positive target for analysis. Glomerular TLR1 and TLR3 immunohistochemical results: 10 non-overlapping glomerular cross-sections were randomly drawn from each histochemical section and the ratio of the positive area of each glomerulus to the entire glomerular area was calculated (in percentage representation), the mean of the ratio of each glomerulus in each slice represents the expression results of TLR1 and TLR3 in the glomerulus of the child. TLR1 and TLR3 immunohistochemical results of renal tubules: 10 nonoverlapping cross-sections of renal tubules were randomly drawn from each histochemical section and the ratio of the positive area of each renal tubule to the area of the entire renal tubule (expressed as a percentage), the mean of the ratio of each renal tubule in the slices represents the expression results of TLR1 and TLR3 in the renal tubules of this child. Statistical Package for the Social Sciences (SPSS) 16.0 software was used for analysis. Measurement data are expressed as mean \pm standard deviation, single-factor analysis of variance is used for comparison between multiple groups and Lysergic acid (LSD) $t$ test is used for pairwise comparison; count data is expressed as percentage or rate and Fisher (F) test is used, $p<0.05$ was considered statistically significant. The expression of TLR1 in kidney tissue is observed. The positive expression of TLR 1 is mainly colored in the cytoplasm, which is brown, granular. In the kidney tissue of the control group, there was a very small amount of TLR1 expression in the glomeruli and tubules and no TLR1 expression in the renal interstitium (fig. 1). In the renal tissue of the PNS group, there was no expression of TLR1 in the renal interstitium. There was no significant difference in the expression of glomerular TLR1 between children with PNS of different pathological types and the control group $(\mathrm{p}>0.05)$. There was a statistically significant difference in the expression of TLR1 in the renal tubules of PNS children with different pathological types and the control group $(\mathrm{p}<0.05)$ (Table 1). The expression of TLR3 in kidney tissue is shown here. The positive expression of TLR3 is also 
mainly cytoplasmic staining and brown, granular. In the kidney tissue of the control group, there is almost no TLR3 expression in the glomeruli and tubules and no TLR3 expression in the renal interstitium (fig. 2). In the renal tissues of the PNS group, there was no expression of TLR3 in the renal interstitium. There was no statistically significant difference in glomerular TLR3 expression between children with PNS of different pathological types and the control group ( $p>0.05)$. The differences in renal tubular TLR3 expression between children with different pathological types of PNS and the control group were statistically significant $(p<0.01)$; among them, four groups of PNS children with different pathological types had higher expression of renal tubular TLR3 than the control group. The MsPGN type was the highest, followed by the MN type, MCD type and FSGS type. The differences between the pairwise comparisons were statistically significant $(\mathrm{p}<0.05)$ (Table 2). TLRs are pathogen recognition receptors that have received much attention in recent years. They are widely distributed, mainly in neutrophils, monocytes, macrophages, dendritic cells and B lymphocytes and other professional immune cells. Recent studies have found that TLRs can also be expressed in kidney cells ${ }^{[7]}$ such as TLR1 which can be expressed in renal tubular epithelial cells, mesangial cells and podocytes etc.; TLR3 can be expressed in kidney macrophages in a model of lupus nephritis. Among them at least 11 types of TLRs were discovered in humans and they are divided into extracellular receptors (TLR1, 2, 4, 5, 6), and intracellular receptors (TLR3, 7, 8, 9). As an extracellular type of TLR1, it mainly recognizes bacterial LPS and PAMPs related to LPS. After TLR1 binds to it, it activates the nuclear factor kappa light chain enhancer of activated B cells (NF-кB) through two signal transduction pathways that are dependent on myeloid differentiation factor 88 (MyD88) and/or independent of MyD88, which in turn causes the transcription of a series of inflammatory factors, such as TNF- $\alpha$, IL-1, IL-8, etc. These inflammatory factors can increase the permeability of the glomerular basement membrane by changing the sulfide metabolism of the glomerular basement membrane in mice, thereby participating in the formation of proteinuria ${ }^{[8]}$. As an intracellular type of TLR3, it mainly recognizes viral RNA, relies on the MyD88 pathway for signal transduction, causes the expression of pro-inflammatory cytokines and initiates and participates in the adaptive immune response ${ }^{[9,10]}$. TLR1 and TLR3 are involved in the formation of many kidney diseases. For example, in the active phase of Immunoglobulin A (IgA) nephropathy, the expression of TLR1 in peripheral blood mononuclear cells increases ${ }^{[11]}$ and the antibody IgA deposited in the kidney can stimulate the proliferation and activation of mesangial cells through the TLR1 signal transduction pathway ${ }^{[12]}$. It suggests that TLR1 mediates immune abnormalities in IgA nephropathy. Infection is an important trigger factor for the onset, recurrence or aggravation of MCD. In 1996, Gelberg proposed that bacterial infection can change the surface charge of podocytes and lead to the formation of proteinuria. There are TLR 2, 3, 4, 9 expressions on podocytes cultured in vitro ${ }^{[13-15]}$. When microbial infection or IL-13 released by T lymphocytes stimulate podocytes, the expression of costimulatory molecule B7.1 (Cluster of Differentiation 80 (CD80)) can be up-regulated through the TLRs signaling pathway and the continuous expression of CD80 can promote podocyte actin reorganization and rupture. Abnormal expression of membrane protein will eventually cause foot process fusion and massive proteinuria $^{[13]}$. Studies have found that TLR3 can play an important role in the occurrence and development of systemic lupus erythematosus by causing the generation of anti-RNA antibodies ${ }^{[16]}$. In this study, immunohistochemical methods were used to observe the expression of TLR1 and TLR3 in renal tissues and normal renal tissues of children with PNS. The results showed that the expression of TLR1 and TLR3 in the renal tubules of children with PNS was significantly increased, while the expression of TLR 1 and TLR3 in the glomeruli and renal interstitium did not change significantly. In addition, TLR1 is most prominently expressed in MCD pathological types and TLR3 is most notably expressed in MsPGN pathological types. It can be inferred from this that TLR1 and TLR3 may play a role in the pathogenesis of PNS in children with different pathological types. According to the main ligand types recognized by TLR1 and TLR3, it is suggested that both bacterial and viral infections may be involved in the pathogenesis of PNS in children. In summary, the pathogenesis of PNS in children is more complicated. Different kidney pathological types may have different mechanisms and there may be multiple factors involved in the pathogenesis of each kidney pathological type. This study only observed the increased expression of TLR 1 and TLR3 in the renal tubules of children with PNS, but whether they are the primary triggers of the pathogenesis of PNS or a result of nephropathy, it needs to be further confirmed and whether they really participate in the pathogenesis of PNS also needs further research. 

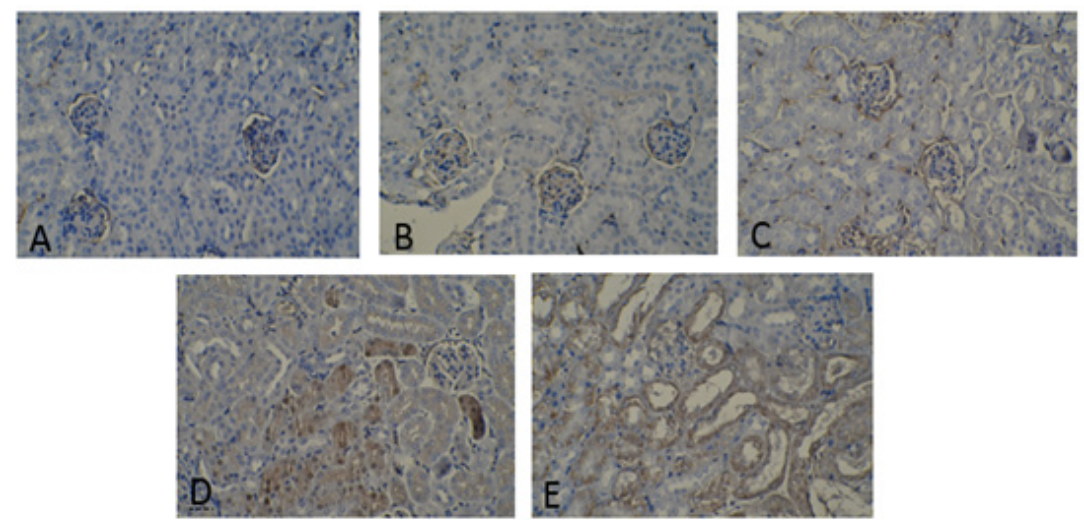

Fig. 1: The expression of TLR1 in kidney tissue (200×), (A) Normal kidney tissue; (B) FSGS; (C) MsPGN; (D) MN; (E) MCD

\section{TABLE 1: TLR1 EXPRESSION IN KIDNEY TISSUE}

\begin{tabular}{lccc}
\hline Group & Number of cases & Glomerulus (\%) & Renal tubules (\%) \\
\hline Control group & 20 & $0.824 \pm 0.18$ & $0.79 \pm 0.29$ \\
MCD & 40 & $1.04 \pm 0.05$ & $87.67 \pm 4.68$ \\
MN & 20 & $1.12 \pm 0.34$ & $58.78 \pm 5.84$ \\
MsPNG & 30 & $1.82 \pm 0.22$ & $26.34 \pm 3.24$ \\
FSGS & 30 & $1.07 \pm 0.24$ & $12.40 \pm 2.63$ \\
F & & 0.67 & 2201.62 \\
p & & 0.428 & 0.000 \\
\hline
\end{tabular}
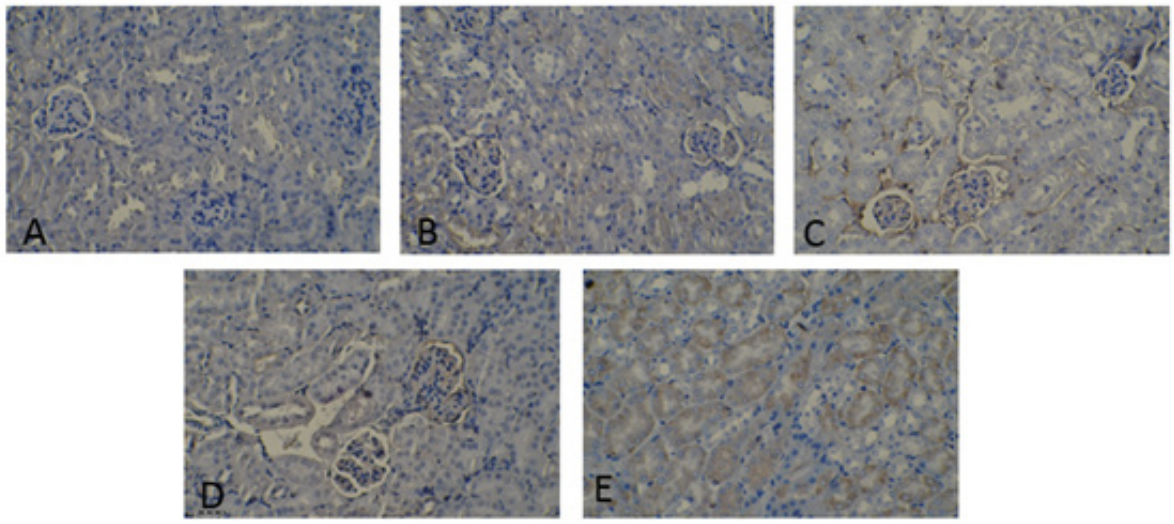

Fig. 2: Expression of TLR3 in kidney tissue (200×), (A) Normal kidney tissue; (B) FSGS; (C) MCD; (D) MN; (E) MsPGN

TABLE 2: TLR3 EXPRESSION IN KIDNEY TISSUE

\begin{tabular}{lccc}
\hline Group & Number of cases & Glomerulus $(\%)$ & Renal tubules (\%) \\
\hline Control group & 20 & $0.56 \pm 0.07$ & 0.32 \\
MCD & 40 & $2.52 \pm 0.43$ & 18.26 \\
MN & 20 & $2.24 \pm 0.32$ & 34.42 \\
MSPNG & 30 & $1.82 \pm 0.26$ & 70.71 \\
FSGS & 30 & $2.07 \pm 0.29$ & 15.95 \\
F & & 1.22 & 401.89 \\
p & & 0.428 & 0.000 \\
\hline
\end{tabular}




\section{Conflicts of interest:}

The authors declared no conflict of interest.

\section{REFERENCES}

1. Tektonidou MG. Antiphospholipid syndrome nephropathy: from pathogenesis to treatment. Front Immunol 2018;9:1181.

2. Rheault MN. Nephrotic and nephritic syndrome in the newborn. Clin Perinatol 2014;41(3):605-18.

3. Zhang L, Yan J, Yang B, Zhang G, Wang M, Dong S, et al. IL-23 Activated $\gamma \delta$ T cells affect Th17 cells and regulatory T cells by secreting IL-21 in children with primary nephrotic syndrome. Scand J Immunol 2018;87(1):36-45.

4. Shalaby SA, Al-Edressi HM, El-Tarhouny SA, El-Bab MF, Zolaly MA. Type 1/type 2 cytokine serum levels and role of interleukin-18 in children with steroid-sensitive nephrotic syndrome. Arab J Nephrol Transplant 2013;6(2):83-8.

5. Dessing MC, Kers J, Damman J, Leuvenink HG, van Goor H, Hillebrands JL, et al. Toll-like receptor family polymorphisms are associated with primary renal diseases but not with renal outcomes following kidney transplantation. PLoS One 2015;10(10):e0139769.

6. Patole PS, Pawar RD, Lech M, Zecher D, Schmidt H, Segerer $\mathrm{S}$, et al. Expression and regulation of Toll-like receptors in lupus-like immune complex glomerulonephritis of MRL-Fas (lpr) mice. Nephrol Dial Transplant 2006;21(11):3062-73.

7. Ren Q, Cheng L, Yi J, Ma L, Pan J, Gou SJ, et al. Toll-like Receptors as Potential Therapeutic Targets in Kidney Diseases. Curr Med Chem 2020;27(34):5829-54.

8. Eleftheriadis T, Lawson BR. Toll-like receptors and kidney diseases. Inflamm Allergy Drug Targets 2009;8(3):191-201.

9. Yang HY, Huang SM, Lu KC, Wu CC, Kang CY, Lin YF, et al. A functional polymorphism in the promoter region of TLR3 is associated with susceptibility to end-stage renal disease. Am J Nephrol 2014;40(2):131-9.
10. Mishra OP, Kumar R, Narayan G, Srivastava P, Abhinay A, Prasad R, et al. Toll-like receptor 3 (TLR-3), TLR-4 and CD80 expression in peripheral blood mononuclear cells and urinary CD80 levels in children with idiopathic nephrotic syndrome. Pediatr Nephrol 2017;32(8):1355-61.

11. Lee JS, Park HK, Suh JS, Hahn WH, Kang SW, Park HJ, et al. Toll-like receptor 1 gene polymorphisms in childhood IgA nephropathy: a case-control study in the Korean population. Int J Immunogenet 2011;38(2):133-8.

12. Gao J, Wei L, Wei J, Yao G, Wang L, Wang M, et al. TLR1 polymorphism rs4833095 as a risk factor for IgA nephropathy in a Chinese Han population: A case-control study. Oncotarget 2016;7(50):83031.

13. Shimada M, Ishimoto T, Lee PY, Lanaspa MA, Rivard CJ, Roncal-Jimenez CA, et al. Toll-like receptor 3 ligands induce CD80 expression in human podocytes via an NF- $\kappa$ B-dependent pathway. Nephrol Dial Transplant 2012;27(1):81-9.

14. Peng Y, Zhang X, Wang Y, Li S, Wang J, Liu L. Overexpression of toll-like receptor 2 in glomerular endothelial cells and podocytes in septic acute kidney injury mouse model. Ren Fail 2015;37(4):694-8.

15. Masum MA, Ichii O, Hosny Ali Elewa Y, Nakamura T, Otani Y, Hosotani M, et al. Overexpression of toll-like receptor 9 correlates with podocyte injury in a murine model of autoimmune membranoproliferative glomerulonephritis. Autoimmunity 2018;51(8):386-98.

16. de Groof A, Ducreux J, Vidal-Bralo L, Tyteca D, Galant C, Marot L, et al. Toll-like receptor 3 increases antigenpresenting cell responses to a pro-apoptotic stimulus, yet does not contribute to systemic lupus erythematosus genetic susceptibility. Clin Exp Rheumatol 2020;38(5):881-90.

This is an open access article distributed under the terms of the Creative Commons Attribution-NonCommercial-ShareAlike 3.0 License, which allows others to remix, tweak, and build upon the work non-commercially, as long as the author is credited and the new creations are licensed under the identical terms

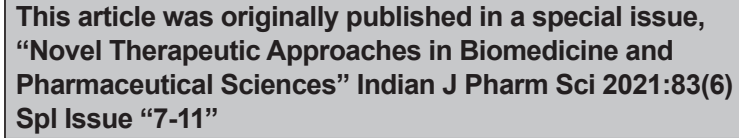

